\title{
Acute disseminated encephalomyelitis following inactivated influenza vaccination in the Brazilian Amazon: a case report
}

\author{
Solange Dourado de Andrade ${ }^{[1],[2],}$ Maria Graciede Filha Santarém Andrade ${ }^{[1],[2]}$, \\ Pablo José Santos ${ }^{[1]}$, Maria de Lourdes Galvão $0^{[1]}$, Mariana Martins de Barros ${ }^{[1]}$, \\ Rajendranath Ramasawmy ${ }^{[1],[3],}$ Izabella Picinin Safe ${ }^{[1]}$, Wuelton Marcelo Monteiro ${ }^{[1],[3],}$ \\ Meritxell Sabidó ${ }^{[1],[4]}$ and Maria das Graças Costa Alecrim ${ }^{[1],[3]}$
}

[1]. Fundação de Medicina Tropical Doutor Heitor Vieira Dourado, Manaus, Amazonas, Brasil. [2]. Centro de Referência para Imunobiológicos Especiais, Manaus, Amazonas, Brasil. [3]. Escola Superior de Ciências da Saúde, Universidade do Estado do Amazonas, Manaus, Amazonas, Brasil. [4]. TransLab. Departament of Medical Sciences. Faculty of Medicine. Universitat de Girona, Spain.

\begin{abstract}
Here, we describe a case of acute disseminated encephalomyelitis (ADEM) that occurred during a plausible risk interval following inactivated influenza vaccination in a previously healthy 27-year-old man from Manaus, Brazil. He was treated with intravenous methylprednisolone and immunoglobulin. One-month follow-up revealed resolution of the brain lesions, but not of the spinal cord lesions. No recurrence or progression of the main neurological symptoms was observed. After two years of monitoring, the patient continues to experience weak lower limbs and urinary retention. Thus, we recommend that ADEM should be considered in a patient presenting with neurological symptoms after influenza vaccination.
\end{abstract}

Keywords: Acute disseminated encephalomyelitis. Inactivated influenza vaccination. Brazilian Amazon.

\section{INTRODUCTION}

Annual influenza vaccination is an important strategy for preventing seasonal influenza virus infections and their potentially severe complications, including death. Vaccination reduces the likelihood of acquiring and transmitting influenza infections ${ }^{(1)}$. The annual inactivated influenza vaccine (IIV) is safe to administer as an injection to both children and adults ${ }^{(2)}$. Minor side effects include self-limited reactions at the injection site such as pain, redness, and swelling ${ }^{(2)}$. Systemic events, such as mild and self-limited fever, malaise, and myalgia, can occur after the IIV has been administered. In some cases, fewer instances of ocular or respiratory symptoms, immediate hypersensitivity, increased risk for febrile seizures in young children, and temporally associated Guillain-Barré Syndrome have been reported ${ }^{(2)}$.

Acute disseminated encephalomyelitis (ADEM) is an inflammatory demyelinating disease of the central nervous system ${ }^{(3)}$. Its onset is acute and often rapidly

Corresponding author: Dra. Solange Dourado de Andrade. Avenida Pedro Teixeira 25, Dom Pedro, 69040-000 Manaus, Amazonas, Brasil.

Phone: 5592 2127-3473

e-mail: douradosol@yahoo.com.br

Received 19 December 2014

Accepted 29 May 2015 progressive $e^{(4)}$. It is traditionally monophasic, but some patients may have recurrences. ADEM typically presents with multifocal neurological signs, including motor, sensory, cranial nerve, and brainstem deficits, as well as nonspecific symptoms such as headache, malaise, and altered mental status (i.e., encephalopathy) $)^{(4)}$. The diagnosis is supported by the presence of one or more supratentorial or infratentorial demyelinating lesions on brain magnetic resonance imaging (MRI) and the absence of destructive black hole lesions on T1-weighted MRI ${ }^{(4)}$. Abnormal cerebrospinal fluid findings such as mild lymphocytic pleocytosis and slightly elevated cerebrospinal fluid (CSF) protein level are suggestive of ADEM, but not essential for diagnosis.

Prior viral infections (measles, smallpox, and chickenpox) are known risk factors, and ADEM has rarely been reported to be in temporal association with vaccinations. The rate of ADEM cases diagnosed following vaccination in a biologically plausible risk interval is as low as $0.1-0.2$ per 100,000 vaccinated individuals of any age ${ }^{(5)}$. However, it is more common in children. ADEM has been reported following measles, mumps, rubella, smallpox, hepatitis B, and $\mathrm{IIV}^{(6)}$. Severe neurological complications including ADEM associated with influenza vaccination have been sporadically reported from countries with a good surveillance system ${ }^{(7-8)}$. Here, we report a case of ADEM following influenza vaccination that occurred in the western Brazilian Amazon. 


\section{CASE REPORT}

We describe a case of neurological complications that occurred during a biologically plausible risk interval following IIV administration in a 27-year-old previously healthy man living in Manaus (State of Amazonas, Brazil). The vaccine was administered six days before symptoms onset as part of the city's immunization campaign in 2012. The patient worked as an administrative staff, had never smoked, and did not report any previous chronic or severe health conditions or infections. There was no travel history or contact with animals or rural areas before the onset of symptoms. He did not show any clear precipitating factors (e.g., infections), and was admitted with symptoms of fever, vomiting, headache, blurred vision, nuchal pain and fluctuating alertness and normal neurological examination. Under suspicion of bacterial meningitis, a broad-spectrum antibiotic (meropenem) was prescribed, but he showed no clinical improvement despite intravenous administration every $8 \mathrm{~h}$ for 14 days. He was hospitalized for 25 days from the day of symptoms onset.

The patient developed progressive weakness in all four limbs eight days post-vaccination. Neurological signs and symptoms progressed until he was unable to walk, and were associated with urinary retention, constipation, and paresthesia over the trunk and legs. The CSF was colorless with 85 white cells/ $\mu \mathrm{L}$, (normal range: 0-5 cells $/ \mu \mathrm{L}), 100 \%$ lymphocytes, $149.82 \mathrm{mg} / \mathrm{dL}$ CSF protein (normal range: 15-60 mg/dL), and $63 \mathrm{mg} / \mathrm{dL}$ glucose (normal range: $50-80 \mathrm{mg} / \mathrm{dL}$ ). His CSF was subjected to Gram staining, Ziehl-Neelsen staining for Mycobacterium tuberculosis, and nankin staining for fungi. The CSF was also subjected to polymerase chain reaction (PCR) to test for Flaviviruses and the results were negative for all (dengue, yellow fever, Ilheus, Rocio, and Saint Louis encephalitis viruses). Fluid-attenuated inversion-recovery (FLAIR) and T2-weighted MRI images revealed brain and spinal cord lesions (Figure 1A and 1B). Thick blood smear was negative for Plasmodium spp. Serology tests were negative for human immunodeficiency virus 1 (HIV-1), viral hepatitis, rubella immunoglobulin M (IgM), and rubella immunoglobulin $\mathrm{G}$ ( $\mathrm{IgG}$ ). Respiratory viruses (adenovirus, influenza $\mathrm{A} 1$, influenza $\mathrm{B}$, parainfluenza, and respiratory syncytial virus) were tested for using a nasopharyngeal swab and all were negative. No antinuclear antibodies, rheumatoid factor, and alpha fetoprotein were detected in the serum.

Cranial MRI showed multiple hyperintense lesions on T2 and FLAIR, and they involved the periventricular and subcortical white matter, as well as large areas of the medulla oblongata and pons and the splenium of the corpus callosum, bilaterally and asymmetrically. Cervical and dorsal spine MRI revealed focal areas of abnormal signal density with contrast enhancement, and these were randomly distributed on the sides of the cervical-dorsal cord that runs from the brainstem-spinal transition to the cone. The joint analysis of the MRI of the brain and spinal cord indicated ADEM. Based on the temporal association, exclusion of alternative etiologies, neurological findings, and MRI displaying diffuse white matter lesions, we diagnosed our patient's condition to be ADEM following influenza vaccination.

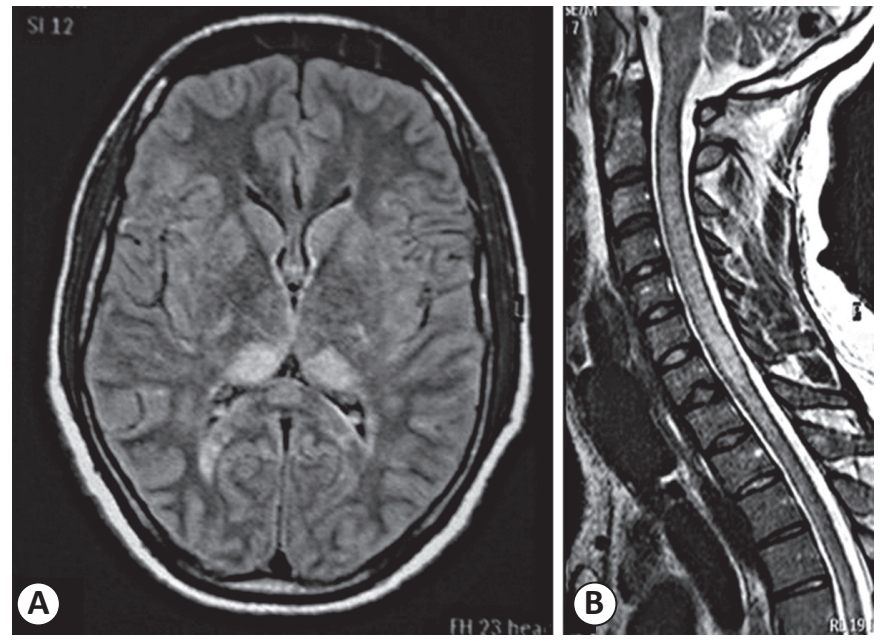

FIGURE 1 - A: FLAIR MRI showing bilateral hyperintensity of the pulvinar nuclei of the thalamus. B: T2-weighted MRI showing diffuse hyperintensity throughout the spinal cord. FLAIR: fluidattenuated inversion-recovery; MRI: magnetic resonance imaging.
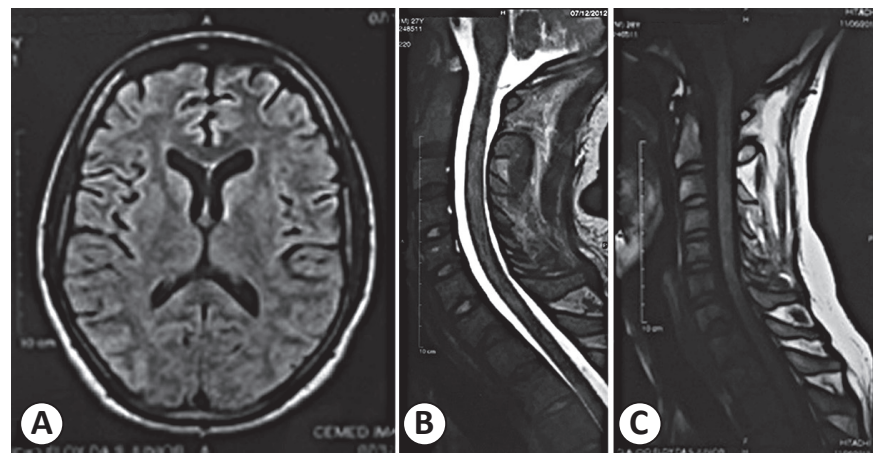

FIGURE 2 - Follow-up using T2-weighted MRI. One month after symptoms initiation, A: FLAIR MRI of the brain showing no alterations. B: Spinal cord MRI showing myelopathy. C: T2-weighted MRI four months after treatment initiation showing resolution of the spinal cord lesions. MRI: magnetic resonance imaging; FLAIR: fluid-attenuated inversion-recovery.

In the hospital, the patient was treated with intravenous methylprednisolone (500 mg daily for five consecutive days), intravenous immunoglobulin $(2,500 \mathrm{mg} / \mathrm{kg}$ daily for five consecutive days), and vitamins B and C. After being discharged, he received a second cycle of intravenous methylprednisolone at the same dose and physiotherapy at home $(2 \mathrm{~h} /$ day for 2 months and $1 \mathrm{~h}$ daily thereafter). The one-month follow-up MRI revealed resolution of the brain lesions (Figure 2A), but not of the spinal cord, which were suggestive of inflammation (Figure 2B). The last spinal MRI performed four months after symptoms onset showed resolution of the lesions (Figure 2C). After two years of monitoring, neurological examination showed the persistence of paraparesis and paresthesia in the lower limbs, and the patient continued to experience weakness in his lower limbs and urinary retention. No recurrence or progression of symptoms was seen, suggesting a monophasic episode that ruled out multiple sclerosis. 


\section{DISCUSSION}

Acute disseminated encephalomyelitis has no pathognomonic clinical features. However, a combination of neurological symptoms in particular temporally associated with an infection or vaccination should alert the clinician of a possible diagnosis of $\mathrm{ADEM}^{(5)}$. Its clinical presentation includes fever, altered consciousness, and multifocal neurological findings. Symptom onset is usually rapid with progression over hours to a peak in days. Focal presentation in ADEM is rather heterogeneous and depends upon the location and degree of demyelination within the central nervous system. MRI is essential for ADEM diagnosis; diffuse or multifocal white matter lesions on FLAIR or T2-weighted sequences are characteristic of $\mathrm{ADEM}^{(4)}$. Lumbar puncture may reveal lymphocytic pleocytosis or elevated protein level. Abnormal CSF is suggestive of ADEM, but not a requisite for diagnosis.

Based on the MRI findings and on excluding acute infective condition, we hypothesized that the IIV might have been related to ADEM in our patient. We then applied the causality criteria to this case, an essential step before diagnosis. According to the World Health Organization (WHO) causality assessment criteria, the association of any event with vaccination is classified as very likely, probable, possible, unlikely, unrelated, or unclassifiable. In the present case, the relationship between influenza vaccination and ADEM would be classified as possible ${ }^{(9)}$. Although the WHO criteria are generally well accepted and widely used, it is important to note that there are significant limitations when applying them to vaccine-related neurological adverse events ${ }^{(10)}$. A neurological event with a reasonable time relationship to IIV administration is automatically classified as possible, despite the fact that this event could be explained by an underlying disease, pathogens, or other drugs. Following clinical evaluation, the relationship between the same event and vaccination would be classified as unlikely because an alternative condition would explain its occurrence ${ }^{(10)}$.

In the most recent review of vaccine-related adverse events conducted by the Institute of Medicine, it was concluded that there is insufficient or lack of epidemiological evidence to either accept or reject a causal association between ADEM and most vaccines, and that the mechanistic evidence based on natural infection is weak ${ }^{(11)}$. This distinction between temporal coincidence and causality is difficult, thus certain causality criteria have been developed. According to the Brighton Collaboration definition, the event observed in the present case was ADEM with level 1 (achieved) certainty ${ }^{(12)}$.

Once ADEM is diagnosed, treatment should begin as soon as possible. Most patients with ADEM improve with treatment, but complete recovery occurs in only $10-46 \%$ patients, with motor deficits and/or cognitive impairment often persisting in the remainder. In fulminant cases, death may occur in $4-12 \%$ patients. Corticosteroids are accepted as first-line therapy ${ }^{(3)}$. For steroid-unresponsive patients, plasma exchange or intravenous immunoglobulin may be used. Poor outcome of treatment is associated with older age, female sex, degree of functional impairment at clinical onset, CSF protein level, spinal cord involvement, peripheral nervous system damage, and poor response to corticosteroids ${ }^{(5)}$. A few patients may either have recurrences that respond to glucocorticoid therapy or are at risk for development of multiple sclerosis.

Our findings underscore the need to consider ADEM when a patient presents with neurological symptoms after influenza vaccination, which is usually administered annually to children and adults in many countries. In our patient, the symptoms and signs compatible with ADEM presented with a reasonable time relationship after IIV administration.

\section{FINANCIAL SUPPORT}

This research was funded by the Fundação de Medicina Tropical Doutor Heitor Vieira Dourado (FMT-HDV), Manaus, State of Amazonas, Brazil.

\section{REFERENCES}

1. Grohskopf LA, Shay DK, Shimabukuro TT, Sokolow LZ, Keitel WA, Bresee JS, et al. Prevention and Control of Seasonal Influenza with Vaccines: Recommendations of the Advisory Committee on Immunization Practices - United States, 2013-2014. MMWR Recomm Rep 2013; 62:1-43.

2. Center of Diseases Control (CDC). Seasonal Influenza Vaccine Safety: A Summary for Clinicians (Internet). Atlanta; 2014. (Cited 2014 November 25). Available at: http://www.cdc.gov/flu/ professionals/vaccination/vaccine_safety.htm

3. Noorbakhsh F, Johnson RT, Emery D, Power C. Acute disseminated encephalomyelitis: clinical and pathogenesis features. Neurol Clin 2008; 26:759.

4. Höllinger P, Sturzenegger M, Mathis J, Schroth G, Hess CW. Acute disseminated encephalomyelitis in adults: a reappraisal of clinical, CSF, EEG, and MRI findings. J Neurol 2002; 249:320.

5. Menge T, Kieseier BC, Nessler S, Hemmer B, Hartung HP, Stüve O. Acute disseminated encephalomyelitis: an acute hit against the brain. Curr Opin Neurol 2007; 20:247-254.

6. Huynh W, Cordato DJ, Kehdi E, Masters LT, Dedousis C. Postvaccination encephalomyelitis: literature review and illustrative case. J Clin Neurosci 2008; 15:1315-1322.

7. Williams SE, Pahud BA, Vellozzi C, Donofrio PD, Dekker CL, Halsey $\mathrm{N}$, et al. Causality assessment of serious neurologic adverse events following 2009 H1N1 vaccination. Vaccine 2011; 29:8302-8308.

8. Machicado JD, Bhagya-Rao B, Davogustto G, McKelvy BJ. Acute disseminated encephalomyelitis following seasonal influenza vaccination in an elderly patient. Clin Vaccine Immunol 2013; 20:1485-1486.

9. Collet JP, MacDonald N, Cashman N, Pless R. Monitoring signals for vaccine safety: the assessment of individual adverse event reports by an expert advisory committee. Advisory Committee on Causality Assessment. Bull World Health Organ 2000; 78:178-185.

10. Kavadas FD, Bitnun A, MacGregor D, Heurter H, Ford Jones EL. Acute neurological events associated with influenza vaccination: are the WHO criteria for assessing causality adequate? Scand J Infect Dis 2008; 40:565-570.

11. National Research Council. Adverse Effects of Vaccines: Evidence and Causality. Washington: The National Academies Press; 2012.

12. SejvarJJ,KohlKS, Bilynsky R, Brighton Collaboration Encephalitis Working Group. Encephalitis. Myelitis, and acute disseminated encephalomyelitis (ADEM): case definitions and guidelines for collection, analysis, and presentation of immunization safety data. Vaccine 2007; 25:5771-5792. 